\title{
Eine Absorptionsmethode zur Herstellung weitgehend spezifischer Anti-Gc-Seren
}

\author{
Von G. BundschuH und U. Krause \\ Aus dem Institut für gericbtliche Medizin der Humboldt-Universität ₹u Berlin (Direktor: Professor Dr. med. O. Prokop)
}

(Eingegangen am 15. Januar 1965)

\begin{abstract}
Es wird eine einfache Absorptionsmethode, mit der Anti-Gc haltige Immunseren weitgehend zu Anti-Gc-Seren spezifiziert werden können, beschrieben. Als Absorptionsmaterial werden hitzeinaktivierte Humanseren verwendet. Während die für die elektrophoretische Wanderung ausschlaggebenden Ladungen bereits durch die Inaktivierung zerstörbar sind, werden die für die Antigen-Antikörper Reaktion in vitro erforderlichen Rezeptoren des Antigens erst durch das zusätzliche Einfrieren unwirksam. Durch Immunisieren mit Kochfiltrat kann dagegen noch Anti-Gc erzeugt werden.
\end{abstract}

A simple absorption method is described whereby anti-Gc-containing immunoserum can be made largely specific to anti-Gc-sera. Heat-inactivated human sera are used as absorption material. While the charges which determine the electrophoretic migration are destroyed by the inactivation, the receptors of the antigens, which are necessary in vitro for the antigen-antibody reaction, are not inactivated until the preparations are also frozen. Upon immunization with koch-filtrate, however, anti-Gc is still producced.

HIRSCHFELD entdeckte 1959 erbliche Varianten eines Alpha $_{2}$-Globulins, die er "Group specific component" nannte. Als Nachweismethode verwendete er die Immunoelektrophorese. Die entsprechenden Präzipitationsbögen liegen im Alpha $a_{2}$-Bereich unmittelbar in Kanalnähe (in den nachfolgenden Abbildungen jeweils durch einen Punkt gekennzeichnet). Während dieses Globulin für die Klinik gegenwärtig noch ohne Bedeutung ist, spielt es in der forensischen Serologie bereits eine besondere Rolle. So können aus einer Elternpaarung mit gleichen reinerbigen Phänotypen nur Nachkommen resultieren, die den gleichen Phänotyp wie die Eltern aufweisen. Abweichungen lassen auf Illegitimität schließen. Die Ausschlußchance eines der Vaterschaft zu Unrecht beschuldigten Mannes beträgt auf Grund der Genfrequenz etwa 17\%. Darin liegt die besondere Bedeutung für die forensische Serologie: - Um die Diagnose des jeweiligen Gc-Typs zu erleichtern und damit auch die Untersuchungstechnik weniger Geübten zugänglich zu machen, wurden von uns Absorptionsmethoden untersucht und die Ergebnisse nachfolgend mitgeteilt.

Die routinemäßige Bestimmung der Gc-Typen in der forensischen Serologie wird für viele Untersucher noch dadurch erschwert, daß nicht genügend Antiseren in ausreichender Qualität zur Verfügung stehen. Viele Untersucher stellen sich die Antiseren durch Tierimmunisierung selbst her. Als Mittlertiere werden meist Kaninchen verwendet. Auch die erfolgreiche Immunisierung von Ziegen wurde beschrieben (1).

Nach unseren Erfahrungen bilden etwa 70\% der immunisierten Kaninchen ein umfangreiches Anti-Menschspektrum, aber nur etwa die Hälfte dieser Tiere bildet zugleich ein brauchbares Anti-Gc. In den übrigen Antiseren ist die Anti-Gc-Komponente entweder zu schwach oder wird durch andere Linien überdeckt. $\mathrm{Da}$ eine elektive Anfärbung der Gc-Komponente gegenwärtig nicht bekannt ist, so ist selbst bei technisch einwandfreier Ausführung der Immunoelektrophorese in einigen Fällen der Gc-Typ nicht mit genügender Sicherheit bestimmbar. Auch der von HrRSCHFELd (2) angegebene Verifikationstest führt nicht immer zum Ziel, insbeson- dere dann nicht, wenn das nicht sicher bestimmbare Serum dem Typ Gc 2-1 angehört. Aus unserem Routinematerial seien einige Fälle aufgeführt, die die Schwierigkeit einzelner Bestimmungen dokumentieren.

In der Abbildung 1 werden die Auftrennungen zwei verschiedener Serumproben wiedergegeben, von der lediglich die Serumprobe Nr. 1 eindeutig als Gc 1-1 erkennbar ist. Die Serumprobe Nr. 2 gehört - wie mit anderen Antiseren ermittelt wurde - dem Typ Gc 2-1 an. Aus der hier gezeigten Auftrennung ergibt sich der Verdacht, daß die Spornbildungen über dem Plateau des Gc-Bogens zwei bisher nicht bekannte Partialantigene des Gc-Protein sanzeigen. Diese Spornbildungen wurden nur bei den Gc-2-1-Typen beobachtet. Das verwendete Antiserum wurde unter 145 verschiedenen Anti-GcSeren von Kaninchen im Verlaufe von 3 Jahren zweimal beobachtet. Möglicherwèise wurden diese Spornbildungen bei anderen Antiseren früher übersehen. Durch eine Absorption soll überprüft werden, ob die im GcBèreich mit angezeigten Antigene Gc-Spezifität besitzen, also Partialantigene dieses Proteins sind, oder sich zufällig in dieser Position darstellen.

In der Abbildung 2 wird die immunoelektrophoretische Auftrennung von zwei anderen Humanseren gezeigt. Als Anti-Gc wurde ein Serum vom Kaninchen $\mathrm{K}_{6}$ verwendet. Während die Serumprobe Nr. 42 eindeutig als

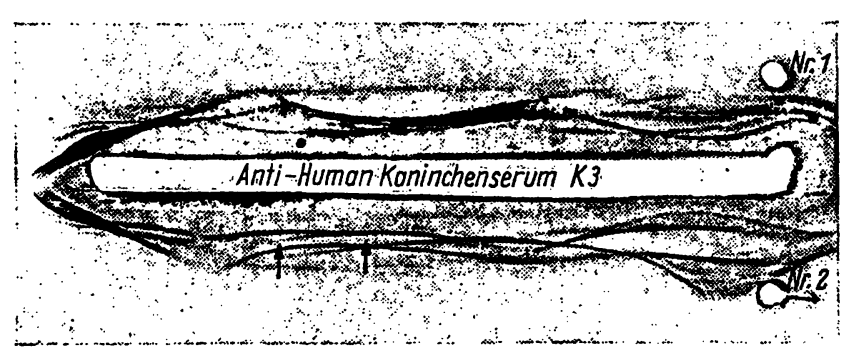

Abb. 1

Die Serumprobe Nr. 2 weist über dem Plateau des Gc-Bogens (Gc 2-1) Spornbildungen auf (durch Pfeile gekennzeichnet).

$\mathrm{Ob}$ es sich um Partialantigene der Gc-Komponenten Gc1 und Gc 2 handelt, läßt sich aus der Auftrennung nicht zweifelsfrei erkennen. Diese Spornbildungen wurden nur bei Gc2-1 Typen beobachtet 


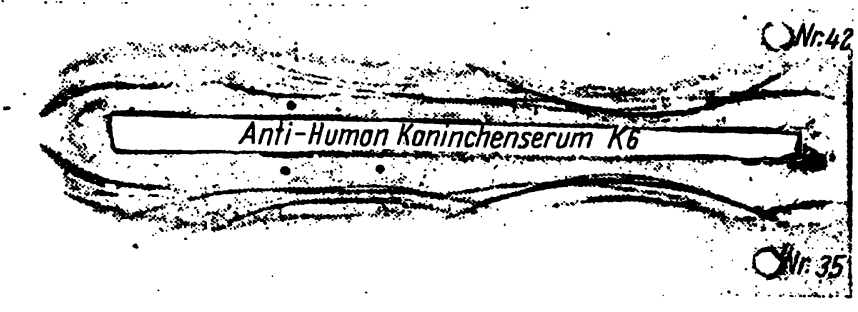

Abb. 2

Die Serumprobe Nr. 35 ist nach dieser Auftrennung in bezug auf den Gc-Typ nicht sicher zu beurteilen. Sehr wahrscheinlich liegt der Typ Gc 2-2 vor (Hp 1-1), vgl. Abb. 3

Gc 1-1 bestimmt wurde, war der Typ der Serumproben Nr. 35 nicht erkennbar. Diese Serumprobe stammte von einem $1 \mathrm{Jahr}$ alten Mädchen und wurde durch Eilpost zur Untersuchung gesandt. Bei der Bestätigung eines durch Voruntersucher angenommenen Types Gc 2-2 würde eine Ausschlußkonstellation resultieren. Durch die Verwendung eines anderen Antiserums (Anti-Gc-Serum vom Pferd; Pasteur-Institut, Paris) konnte die Diagnose ebenfalls nicht eindeutig gestellt werden. Nach dieser Auftrennung hätte die Diagnose Gc1-1 oder Gc 2-2 lauten können (s. Abb.3). Das Phänomen einander kreuzender Präzipitationsbögen im Gc-Bereich (Abb. 3; Gc 1-1 oder Gc 2-2) ist häufiger zu beobachten. Einer der beiden Bögen entspricht in der Regel dem Haptoglobin. Mit einem absorbierten, spezifisch reinen Anti-Gc-Serum wäre die Diagnose hier sicher möglich gewesen. Zur Zeit der Untersuchung des Serums stand uns absorbiertes Antiserum noch nicht zur Verfügung.

Cleve und Mitarbeiter (3) beschrieben 1963 zwei bisher unbekannte Gene, die Gc-Phänotypen steuern: Gc Cbip und Gc $A b$. Die aus der Kombination der nunmehr vier bekannten Gene resultierenden Phänotypen dürften sich in der Immunoelektrophorese in einigen Fällen sehr schwer gegeneinander abgrenzen lassen. Auch in derartigen Fällen könnte ein spezifisch absorbiertes Anti-Gc-Serum von Vorteil sein.

Um unseren Bedarf an Anti-Gc-Serum zu decken, wurden im Verlaufe von 3 Jahren 11 Pferde mit gepooltem

Nr. 42

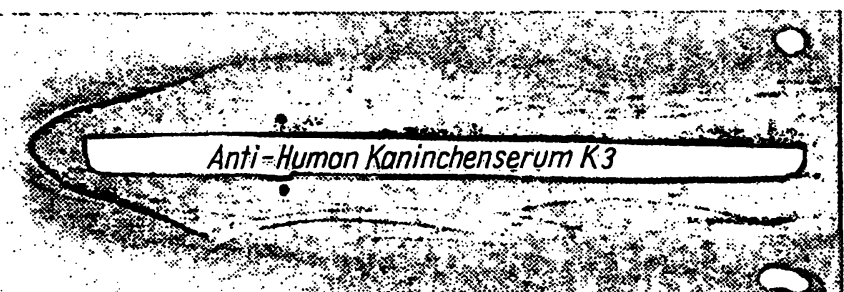

Nr. 42

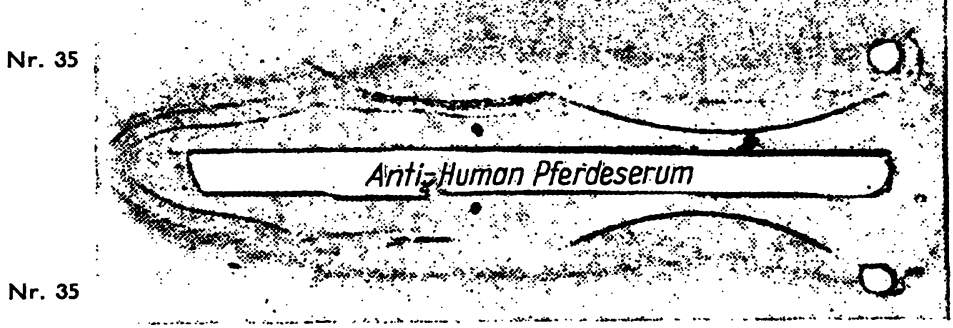

Abb. 3

Auftrennung der Serumprobe Nr. 35 mit einem anderen Antiserum (Anti-Humanserum vom Pferd; Pasteur-Institut, Paris)
Humanserum und Seren von Einzelspendern immunisiert. Von den immunisierten Pferden bildeten 2 Tiere ein bedingt brauchbares Anti-Gc. Unsere Erfahrungen über die Immunisierung von Pferden werden an anderer Stelle veröffentlicht. Das präzipitierende Anti-Humanserum des Versuchspferdes Nr. 10, das auch zugleich Anti-Lp gebildet hat (BundschuH, 1964), zeigte in unabsorbiertem Zustand die Gc-1-1-Typen sehr deutlich an; die Typen Gc 2-1 und 2-2 waren dagegen schwer erkennbar, so daß anfangs an die Möglichkeit einer spezifischen Digität dieses Antiserums für den Typ Gc 1-1 gedacht wurde. Wie sich aber später herausstellte, wurde das Präzipitat der Gc-Typen 2-1 und 2-2 durch das Präzipitat des Hp-Komplexes überdeckt, das bei diesem Antiserum annähernd die gleiche Position wie das Gc einnimmt. In Abbildung 4 wurde das Rohserum dieses Pferdes verwendet.

Der Typ Gc 2-1 (Abb. 4 unteres Startloch) ist gerade erkennbar. Eine Verdünnung des Antiserums mit NaClLösung erbrachte keine besseren Resultate. Das von REINSKou (4) angegebene Verfahren der weiteren Auftrennung der Serumproben (Entfernung AlbuminStartloch $=8 \mathrm{~cm}$ ), bei dem die Gc-1-1-Typen durch zwei ineinander übergehende und die Gc-2-1-Typen durch drei ineinander übergehende Bögen charakterisiert werden, fühtte mit diesem Antiserum in einigen aber nicht in allen Fällen zum Erfolg. Um dieses Antiserum für die Routine brauchbar zu machen, mußte nach einem Absorptionsverfahren gesucht werden, mit dem die störenden Antikörperkomponenten weitgehend eliminiert werden konnten. Bei der Suche nach einer geeigneten Methode mußte zugleich Wert darauf gelegt werden, daß das entsprechende Absorptionsmaterial billig, in ausreichender Menge und leicht verfügbar ist. Die Absorption mit gereinigten Serumfraktionen, wie sie HIRSCHFELd (5) anwendete (Albumin und Properdin) schied somit aus $\left.{ }^{1}\right)$.

Es wurde versucht, Körperflüssigkeiten oder Organextrakte aus Leichenmaterial zu gewinnen, die kein Gc wohl aber andere Serumeiweißkomponenten enthalten. In Analogie zu einer früheren Untersuchung bezüglich des $\mathrm{Hp}$-Gehaltes in extravasalen Flüssigkeiten (Bund-

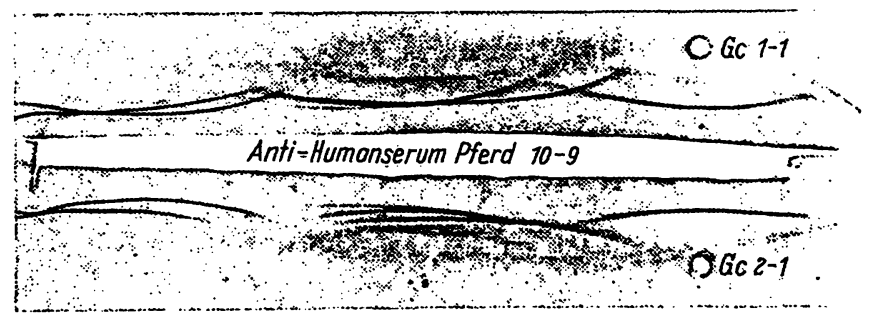

Abb. 4

Auftrennung zwei verschiedener Humanseren Gc $1-1$ und Gc2-1. Das verwendete Immunserum stammt vom Pferd: Versuchstier Nr. 10; Entnahmecharge 9. Die Gc-Linien werden im vorliegenden Fall deutlich angezeigt. In der Mehrzahl der Auftrennungen waren die Präzipitationsbögen dex Typen Gc $2-1$ und 2-2 nicht sicher erkennbar

1) Úber eine Methode zur Properdinherstellung wird von ScrürzLER (6) berichtct. 

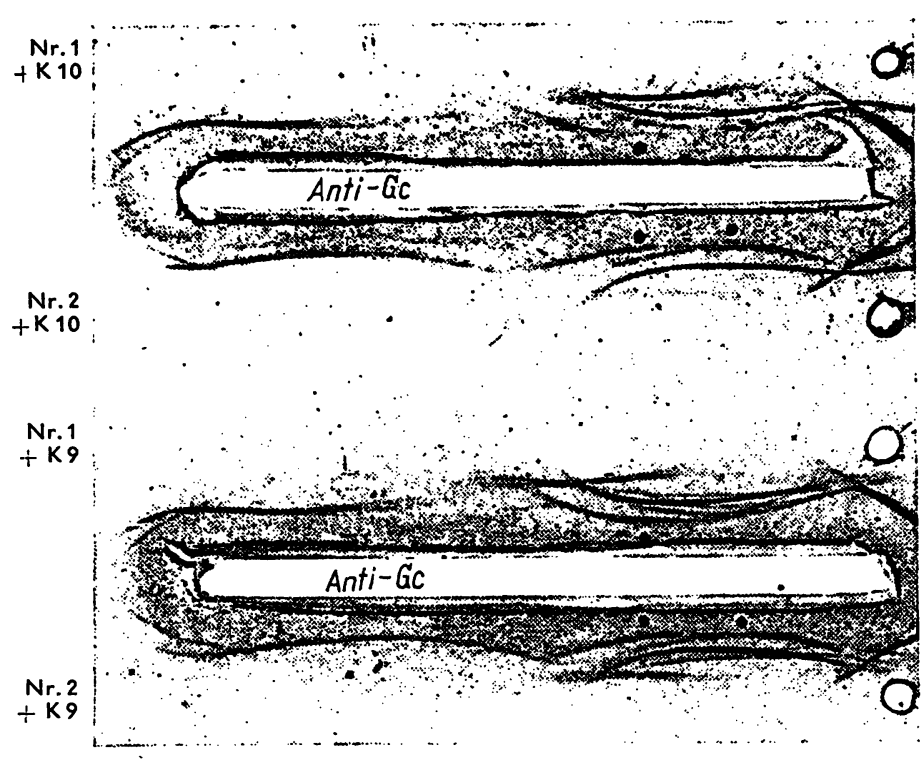

Abb. 5

Die Serumproben Nr. 1 und Nr. 2 wurden vor der immunoelektrophoretischen Analyse mit gleichen Teilen präzipitierenden AntiHumanserums versetzt, das kein Anti-Gc enthielt.

In der oberen Auftrennung wurde präzipitierendes Anti-Humanserum $K_{10}$, in der unteren $K_{9}$ verwendet. In die Antiserumkanäle wurde Anti-Gc-haltiges Immunserum gegeben. Im Alpha ${ }_{1-}$ und teilweise im $\mathrm{Alpha}_{2}$-Bereich wurden einige Serumeiweißkomponenten durch das hinzugegebene Anti-Gc freie Immunserum bereits vor der Elektrophorese präzipitiert und kamen so nicht mehr zur Darstellung

SснUн (7)) wurden verschiedene Körperflüssigkeiten, Extravasate und Organextrakte auf ihren Gc-Gehalt untersucht. Mit der Ausnahme nur einiger Liquores wurde in fast allen anderen Proben - wenn Serumeiweißkörper nachgewiesen werden konnten - auch Gc gefunden. Obwohl die Absorption mit der Cerebrospinalfüssigkeit, die in einigen Fällen kein $\mathrm{Gc}$ enthielt, möglich war, wurde wegen der erheblichen Beschaffungsschwierigkeiten ausreichender Mengen geeigneten Liquors dieser Weg wieder verlassen. Eine zusammenfassende Darstellung der durchgeführten Versuche wird im Rahmen einer Dissertation gegeben (KRAUSE, 1965). $\mathrm{Da}$ uns ausreichende Mengen Immunseren von Kaninchen zur Verfügung standen, die kein Anti-Gc enthielten, aber im Alpha ${ }_{2}$-Bereich mehrere andere Antikörperkomponenten aufwiesen, wurde überprüft, ob durch Zusatz solcher Immunseren zu den zu untersuchenden Humanseren eine bessere Darstellung der Gc-Typen durch die nachfolgende immunoelektrophoretische Analyse erreicht werden konnte. Den Versuchsergebnissen zufolge ist dieser Weg bedingt geeignet aber an ein zweites Antiserum gebunden. Für die Routine schien uns dieser Weg daher ungeeignet (s. Abb. 5).

In weiteren Versuchen wurden Humanseren zur Absorption verwendet, in denen kein $G c$ nachgewiesen werden konnte. In unserem Routinematerial wird ein Fehlen von $\mathrm{Gc}$ in etwa $0,25 \%$ der Fälle beobachtet. $\mathrm{Ob}$ hier genetische Hintergründe vorhanden sind oder rein pathologische Serumveränderungen vorliegen, ist noch ungeklärt. Auch wurde beobachtet, daß einzelne Serumproben, in denen mit einem Antiserum kein Gc nachgewiesen werden konnte, sich mit einem anderen Antiserum dagegen eindeutige Typen erkennen ließen; offenbar spielen auch methodische Fehler hier eine Rolle. In keinem der von uns untersuchten Fälle konnte mit einem derartigen Humanserum eine überzeugende $\mathrm{Ab}$ sorption eines Antiserums erreicht werden.

Eine Absorption des Antiserums durch Nabelblut ist ebenfalls unmöglich, da Nabelblut bereits $\mathrm{Gc}$ enthält. Offenbar setzt die kindliche Gc-Produktion sehr früh ein. HirsChFeld und Lunell (8) fanden unterschiedliche Gc-Typen im mütterlichen und fötalen Blut (25 Wochen alte und $230 \mathrm{~mm}$ lange $Z$ willingsföten).

In weiteren Versuchen wurde mit inaktivierten Humanseren gearbeitet. Die Seren wurden im Wasserbad auf verschiedene Temperaturen erhitzt und anschließend der Nachweis des Gc-Types versucht. Unsere Ergebnisse stimmen mit den von Stöss und PetTrenkofer (9) etmittelten Werten sehr gut überein: Beim Inaktivieren der zu untersuchenden Serumproben über $+62^{\circ}$ für 30 Minuten kann mit Hilfe der Immunoelektrophorese der Gc-Typ nicht mehr bestimmt werden; die entsprechende Bande fehlt im Elektropherogramm. Die Mehrzahl der übrigen Banden ist noch deutlich ausgeprägt (s. Abb. 6). Ähnliche Versuche wurden bereits 1962 von Prokop und Schlesinger durchgeführt (unveröffentlichte Arbeit).

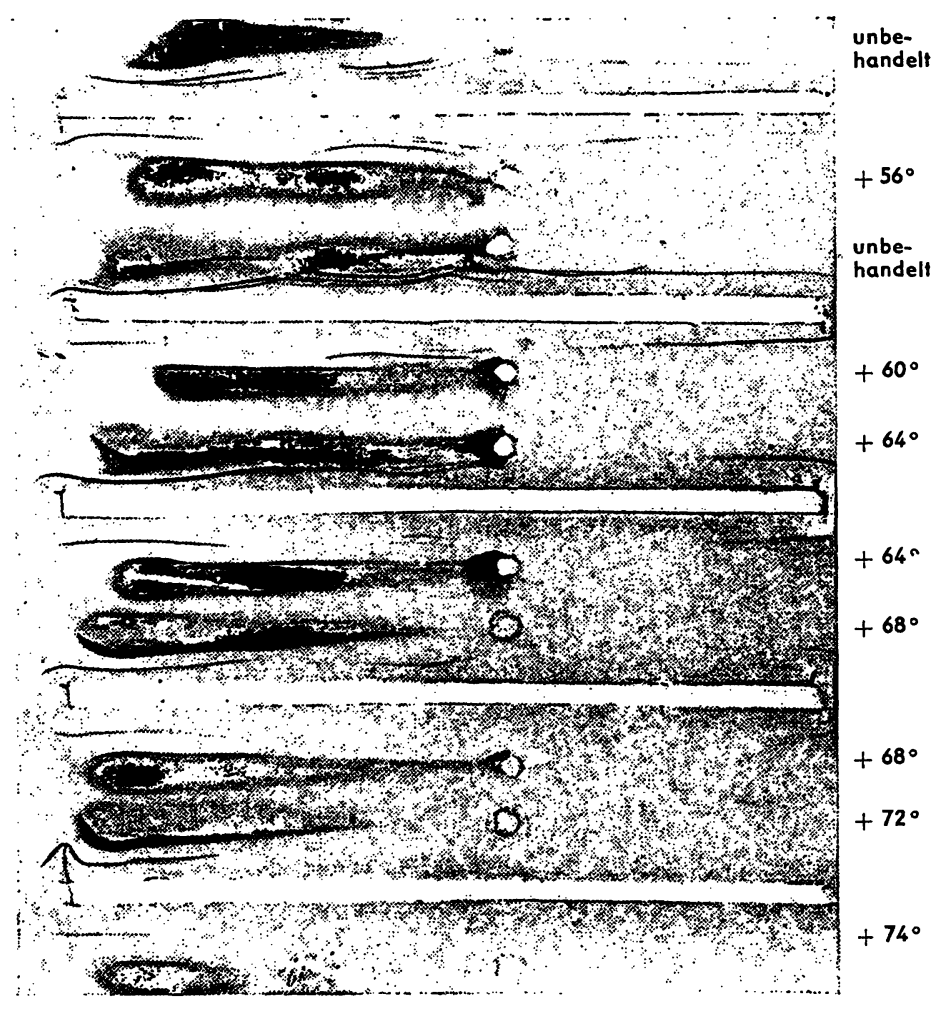

Abb. 6

Für die in der Abbildung dargestellten Inaktivierungsversuche wurden stets Serumproben des gleichen Humanserums(Gc-Typ 2-1) verwendet

Die Inaktivierung erfolgte im Wasserbad über 30 Minuten. Die Anfärbung des Präparates wurde mit Sudanschwarz und anschließend mit Azokarmin vorgenommen 
Mit dem Filtrat gekochter Seren kann aber durch Immunisicrung noch ein Anti-Menschspektrum erzeugt werden. So konnte GiBB an unserem Institut durch Kaninchenimmunisierung mit Kochfiltrat cin Anti-Gc erzeugen. Durch die Immunisierung mit Acetontrockenpulver (Humanserum mit Aceton versetzen, bis ein weißer Niederschlag erfolgt, über Nutschenfilter $G^{3}$ filtrieren, Rückstand zum Immunisieren verwenden) konnte EDER an unserem Institut ein brauchbares AntiGc herstellen (s. Abb. 7). Daraus ist zu ersehen, daß denaturiertes Antigen seine immunologische Reaktionsweise im Tierexperiment weitgehend beibehält. Für hitzedenaturierte Antigene ist- der Ausdruck „Koktoantigene" gebräuchlich. Durch Koktoantigene erzeugte Immunseren werden in der Antigen-Antikörper-Reaktion in vitro sowohl die Koktoantigene als auch die Nativantigene angezeigt. Durch Nativantigene erzeugte Immunseren zeigen dagegen nur die Nativantigene an, nicht das entsprechende Koktoantigen; s. Abbildung 6 (10). Die Thermostabilitätsgrenze ist für die einzelnen Serumeiweißkörper unterschiedlich.

Mit den hitzeinaktivierten Humanseren wurden Absorptionsversuche durchgeführt. Bereits die ersten $E_{r-}$ gebnisse waren positiv.

Hitzeinaktivierte Humanseren werden ebenfalls erfolgreich zum Absorbieren von Antiglobulinseren eingesetzt. Eine Zusammenstcllung der entsprechenden Literatur geben PROKOP und UnLENBRUCK (11). Bemerkenswert ist, daß die gleiche Inaktivicrungstemperatur von einigen Untersuchern angegeben wird: $+62^{\circ}$ bei Sperser (12) $+63^{\circ}$ bei Hunter und ThOmas.

\section{Vorbereitung der Humanseren z!um Absorbieren}

Einzelne oder gepoolte Serumproben (ohne Bcachtung des GcTyps) werden für $30 \mathrm{Min}$. unter mehrmaligem Schütteln auf $+64^{\circ}$ erhitzt. Frische Serumproben können bei diescr Temperatur bereits gelieren. Bei älteren oder gepoolten Scren wurde ein Gclieren bei dieser 'Temperatur nicht beobachtet. Einfricren der erhitzten Serumproben bei etwa $-20^{\circ}$ für 2 bis $3 \mathrm{Std}$, auftauen und zentrifugieren. Den Uberstand (im Vcrhältnis 1 Tcil hitzcinaktiviertes Humanserum und 2 Teilc Antiserum) mit dem zu absorbierenden Immunserum vermischen. Als Absorptionszeit

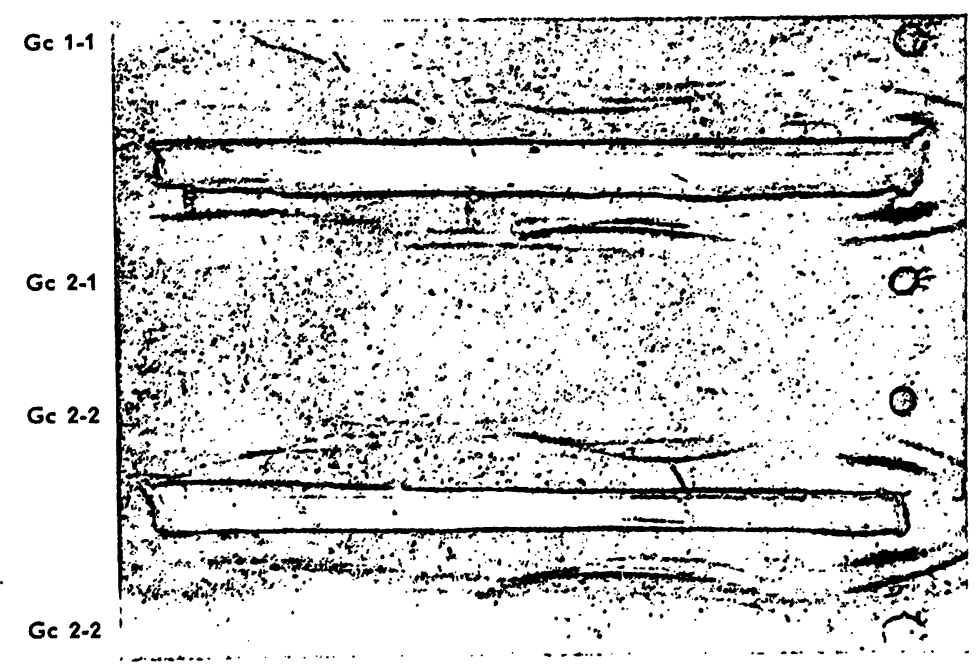

Abb. 7

Das fuir diese Auftrennungen verwendete Immunscrum wurde durch Immunisierung von Kaninchen mit Acetontrockenpulver gewonnen (s. 'Text)

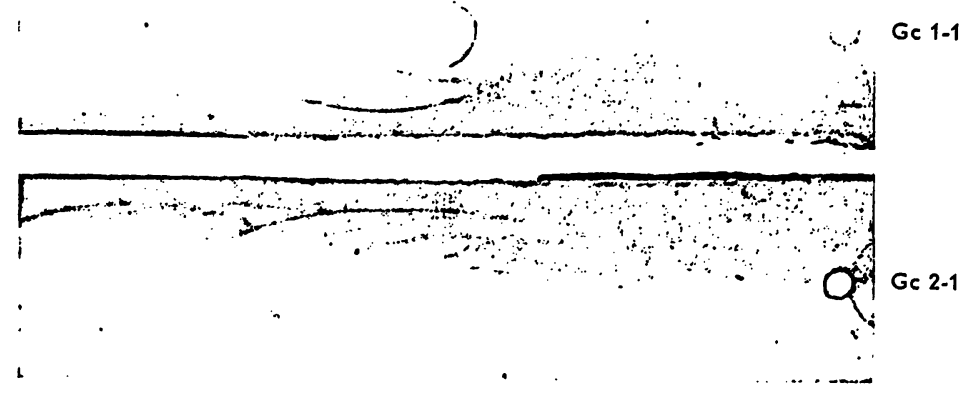

Abb. 8

Für dic Präzipitation wurde das in $\Lambda$ bb. 4 gezcigtc Immunserum vom Pferd 10-9 nach Absorption mit hitzcinaktivicrtem 1-Iumanserum verwendet. Im Alphan $-B$ ereich treten der Gc-Bogen deutlich, der Makroglobulin-u. Hp-Bogen nur andeutungsweisc hervor.
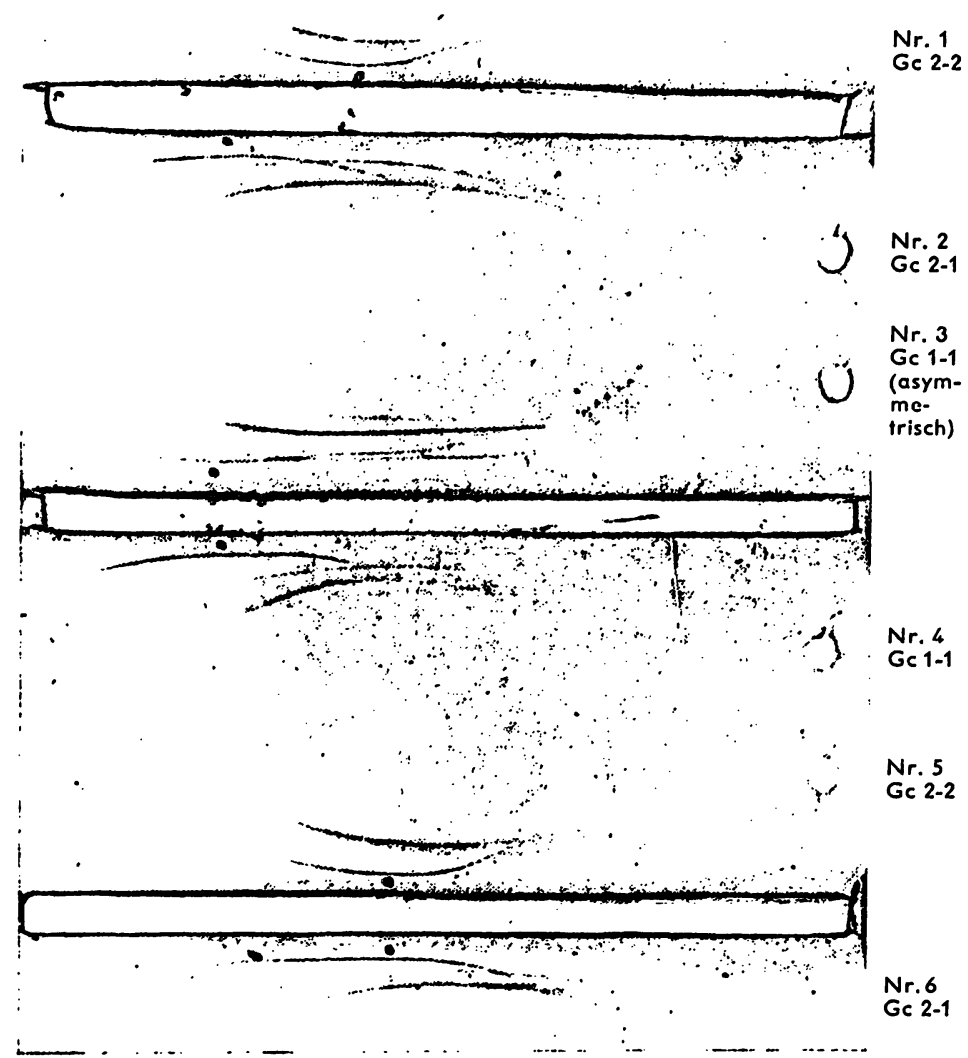

Abb. 9

Auftrennung 6 verschiedener Humanseren \%ur Bestimmung des Gc-Typs. Die Präzipitation erfolgte durch absorbicrtes Immunserum, Pferd 10-9.

Dic asymmetrische Bogenform der Scrumprobe Nr. 3 war nicht reproduzicrbar, es handelt sich offenbar um einen technischen Fehler.

Inaktivierungszeit $30^{\prime}$ bei $64^{\circ}$, nach dem Zentrifugicren 3 Stdn. bei $-20^{\circ}$ eingefroren

reichen nach unscren Erfahrungen bercits 20 Min. bei Zimmertemperatur aus. Die Variation der Absorptionszeit und der Umgebungstemperatur blicb ohne sichtbaren Erfolg.

\section{Untersuclungsergebnisse}

Wurde das frisch absorbierte Antiserum für dic GcBestimmung verwendet, so konnte nach einer Diffusionszeit von etwa $18 \mathrm{Stdn}$. bei $+37^{\circ}$ eine zarte, aber scharfe Gc-Bande bereits in ungefärbtem Zustand der Immuno- 


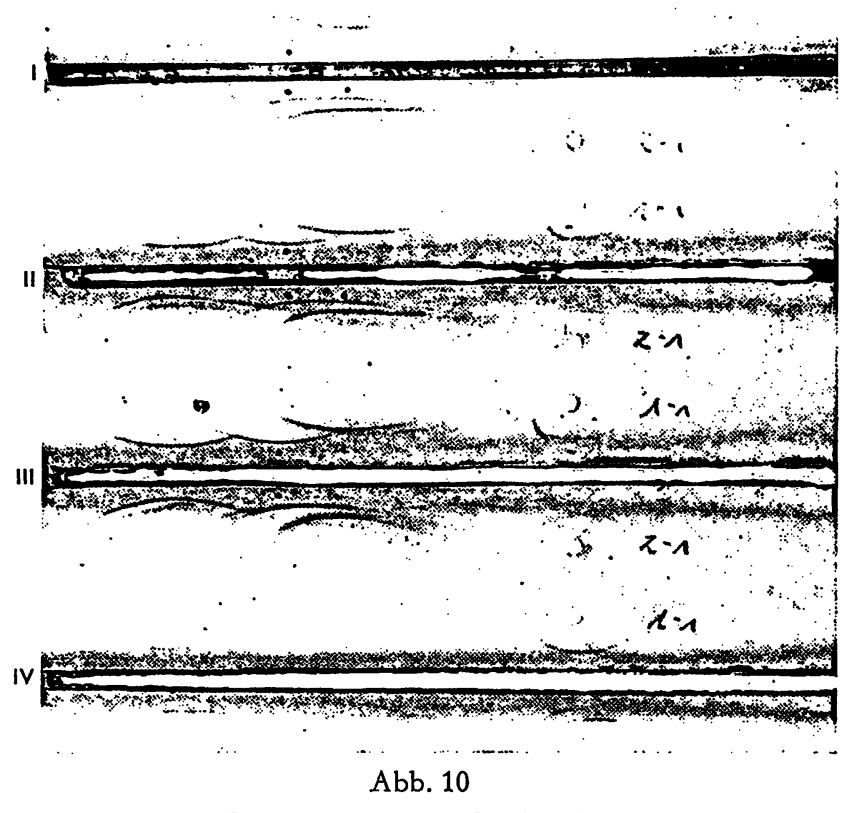

Zur Darstellung der Gc-Typen wurde absorbiertes Immunserum verwendet. Vor der Inaktivierung wurde das Humanserum durch $1 n \mathrm{NaOH}$ auf $\mathrm{pH}=8,6$ eingestellt. Inaktivierungszeit $30^{\prime}$ bei $64^{\circ}$

Kanal I-III: Verschiedene Absorptionszeiten

I $10^{\prime}$ bei Zimmertemperatur III $\mathrm{CO}^{\prime}$ bei Zimmertemperatur II $20^{\circ}$ bei Zimmertemperatur IV $30^{\prime}$ bei Zimmertemperatur jedoch wurde das hitzeinaktivierte Humanserum über Nacht im Tiefkühlschrank belassen

elektrophoresepräparate erkannt werden. Am frischen Präparat ließen sich im Alpha - -Bereich keine weiteren Linien erkennen. Erst nach dem Anfärben des Präparates traten je nach Absorptionsverhältnis weitere Linien schwach hervor, so das Makroglobulin und das Betalipoprotein, ebenso das Alpha ${ }_{1}$-Lipoprotein (s. Abb. 8). Diese Fraktionen stören die Bestimmung der Gc-Typen nicht. Sie sind im Routinetest zur besseren Orientierung erwünscht. - Das Antiserum vom Kaninchen $\mathrm{K}_{3}$, das in unabsorbiertem Zustand bei den Humanseren vom Typ Gc 2-1 über dem Plateau des Gc-Bogen Spornbildungen aufwies (s. Abb. 1), zeigte nach der Absorption nur noch den regulären Gc-Bogen an.

\section{Diskussion}

Durch die von uns beschriebene Absorptionsmethode mit hitzeinaktiviertem Humanserum konnte das im un- absorbierten Zustand für die Gc-Bestimmung nur bedingt brauchbare Immunserum des Pferdes Nr. 10 für die Routine brauchbar gemacht werden. Auch Kaninchen-Antiseren lassen sich mit dieser Methode weitgehend spezifisch zu Anti-Gc absorbieren. Die Versuche zur Lagerung der absorbierten Antiseren sind noch nicht abgeschlossen. - Voraussetzung für den Erfolg ist ein genügend hoher Anti-Gc-Titer im Rohserum.

Grundlage für die Absorption mit hitzeinaktiviertem Humanserum ist die Thermolabilität der Gc-Komponente. Obwohl dieses Verfahren nicht absolut spezifisch ist, da auch andere Komponenten thermolabil sind, ermöglicht es doch eine für Routinezwecke ausreichende Spezifität. - Die Thermolabilität der Gc-Komponente bezieht sich auf die elektrophoretische Mobilität und die Reaktionsweise des Proteins mit dem homologen Antikörper, nicht auf die antigene Wirkung im Tierexperiment: denn wie gezeigt wurde, kann mit gekochtem Serum noch ein Anti-Gc erzeugt werden, das auch mit Nativserum reagiert. Während durch die Hitzedenaturation bereits bei $+62^{\circ}$ am Eiweißmolekül des Gc-Antigens Ladungsveränderungen herbeigeführt werden, die seine elektrophoretische Mobilität verhindern, werden offenbar durch die gleiche Temperatur die Rezeptoren für die Antikörperbildung nur soweit geschädigt, daß sie durch ein Einfrieren auf $-20^{\circ}$ vollends zerstört werden können. Denn wird das hitzeinaktivierte Serum vor der Absorption nicht eingefroren, so wird durch den Absorptionsvorgang auch das Anti-Gc eliminiert. Das Einfrieren ist somit erforderlich, um das Antigen zu zerstören.

Die Ursache für die unterschiedlichen Immunreaktionen mit hitzedenaturiertem Antigen im Tierexperiment und im Diffusionstest bzw. in der Immunoelektrophorese sind sehr wahrscheinlich durch die Löslichkeitsveränderung bedingt, die mit der Denaturierung einhergeht. Während die Löslichkeit des Antigens für den Nachweis durch Elektrophorese und Diffusion notwendig ist, dürfte für die Immunreaktion im Tierexperiment die Löslichkeit keine entscheidende Rolle spielen (Phagozytose des unlöslichen Antigens).

Für die technische Assistenz bei der Durchführung der Arbeit danken wir unserer MTA Fräulein H. MARTH.

\section{Literatur}

1. Kerde, Ch., D. Schlesinger, O. Prokop und A. Vogt, Dtsch. Zschr. "gerichtl. Med., 53, 142 (1963). - 2. Hirschfeld, J., Progr. Allerg. 6, 1 (1962). - 3. Cleve, H., R. L. KIRK, W. C. PARKer, A. G. Bearn, L. E. Schacht, H. Kleinman und W. R. Horsfall, Amer. J. Human. Genetics 15, 368 (1963). - 4. Reinskou, T.: Acta path. mikrobiol. Scand., 59, 4 (1963). Hirschfeld, J.. Science Tools 8, 3 und 17 (1962).-6. Schützler, H., Arch. exper. Vet. med. 17, 877 (1963). - 7. Bundschur, G., I. Menning, H. HANdGe und G. Schubert, Zschr. ärztl. Fort- bild. 56, 14 (1962). - 8. Hirschfeld, J. und N.-O. LunelL, Nature (London) 196, 4860, 1220 (1962). - 9. Sröss, B. und H. J. Pettenkofer, Zbl. Bakt., Abd. I Orig.-Ref. 190, 277 (1963). - 10. Sснмrdт, H., Fortschritte der Serologie. (Erschienen in der Reihe: Wiss. Forschg. Ber. Nat. R.; herausgegeben von LIESEGANG, E.) Steinkopff-Verlag, Dresden und Leipzig (1933). - 11. PROKOP, O. und G. UHLENBRUCK, Lehrbuch der menschlichen Blutund Serumgruppen, I. Aufl. Georg Thieme, Leipzig (1964). - 12. SPEISER, P. und K. Baumgarten, Zschr. Hyg., 141, 151 (1955).
Dr. med. G. Bundschuh Institut für gerichtliche Medizin der Humboldt-Universität zu Berlin Berlin 104, Hannoversche Str. 6 